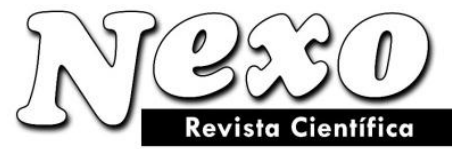

\title{
Morteros a base de vidrio de desecho/escoria de alto horno; activación mecanoquímica del vidrio en soluciones alcalinas.
}

\author{
L. J. Espinoza Pérez ${ }^{1 *}$ J. I. Escalante García ${ }^{2}$ \\ ${ }^{1}$ Universidad Nacional de Ingeniería de Nicaragua \\ e-mail: lester.espinoza@ fiq.uni.edu.ni \\ ${ }^{2}$ Centro de Investigación y de Estudios Avanzados de México \\ e-mail: ivan.escalante@cinvestav.edu.mx
}

(Recibido/received: 22-Octubre-2011; aceptado/accepted: 28-Noviembre-2011)

\section{RESUMEN}

Se investigaron cementos compósitos de escoria de alto horno (EAH) y vidrio de desecho (VD). Una fracción del VD se sometió a tratamientos de activación mecanoquímica (AMQ) para solubilizar $\mathrm{SiO}_{4}$ en una solución alcalina, para obtener soluciones de silicato de sodio (SS) con módulo de sílice mayor que cero y favorecer el desarrollo de resistencia a la compresión. Con las soluciones obtenidas mediante AMQ se prepararon compósitos con 0, 25, 50, 75 y $100 \%$ VD como cementante, y como referencia se utilizaron cementos 100\%EAH con activación alcalina convencional. La resistencia a la compresión de estos últimos disminuyó al aumentar el contenido de $\mathrm{Na}_{2} \mathrm{O}$ y disminuyó con el curado a $60^{\circ} \mathrm{C}$. En contraste, los cementos $100 \% \mathrm{VD}$ requirieron condiciones químicamente más agresivas $\left(12 \% \mathrm{Na}_{2} \mathrm{O}\right.$ y curado a $\left.60^{\circ} \mathrm{C}\right)$ para obtener buenas propiedades mecánicas. Los cementos compósitos con 25 y $50 \% \mathrm{VD}$ requirieron condiciones de activación similares a los cementos 100\%EAH, mientras que aquellos con $75 \%$ VD requirieron condiciones de activación similares a los cementos $100 \%$ VD.

Palabras claves: vidrio de desecho, escoria de alto horno, activación mecanoquímica

\begin{abstract}
Composite cements of Blast Furnace Slag (EAH) and Waste Glass (WG) were investigated. A fraction WG was subjected to mechanochemical activation aiming to solubilize its $\mathrm{SiO}_{4}$ to obtain solutions of sodium silicate (SS) with silica modulus greater than zero to improve the compressive strength of the cements. With the solutions obtained by $\mathrm{AMQ}$, composites of 0 to $100 \%$ WG were prepared, and as referred to cements $100 \%$ BFS with standard alkaline activation. The strength of the latter decreased after increasing the $\mathrm{Na}_{2} \mathrm{O}$ content and decreased when the curing temperature was increased to $60^{\circ} \mathrm{C}$. In contrast, $100 \% \mathrm{WG}$ cements required very aggressive chemical activation conditions $\left(12 \% \mathrm{Na}_{2} \mathrm{O}\right.$ at $\left.60^{\circ} \mathrm{C}\right)$ to obtain good mechanical properties. The composites with 25 and 50\% WG required activation conditions similar to those 100\% EAH cements, while those cements with $75 \%$ WG required activation conditions similar to those of $100 \%$ WG.
\end{abstract}

Keywords: waste glass, blast furnace slag, mechanochemical activation.

\footnotetext{
* Autor para la correspondencia
} 
Espinoza L. y Escalante J.

\section{INTRODUCCIÓN}

El concreto de cemento portland (CP) es el material de construcción más utilizado en el mundo para la construcción de edificios e infraestructura. Sin embargo, la producción de $\mathrm{CP}$ es de alto consumo de combustibles fósiles y de una gran emisión de contaminantes ( 0.85-1 $\mathrm{kg}$ de $\mathrm{CO}_{2}$ emitido a la atmósfera por cada $1 \mathrm{~kg}$ de $\mathrm{CP}$ producido), pues se deben alcanzar temperaturas de clinquerización de $1450^{\circ} \mathrm{C}\left[{ }^{1}\right]$.

Debido a las restricciones ambientales impuestas, la tendencia es tomar medidas como el uso de combustibles y materias primas alternativas, la reducción en el contenido del clínquer, el secuestro del $\mathrm{CO}_{2}$ mediante tratamientos físico-químicos, entre otras.

En Iberoamérica el costo del CP es relativamente alto, lo que conduce a la búsqueda de ahorros mal entendidos que conllevan a degradar la calidad de los materiales de construcción. Por ejemplo, en Nicaragua las normas establecen que por cada $42.5 \mathrm{~kg}$ de CP se deben fabricar 28 bloques de concreto, sin embargo, se llegan a producir hasta 50 bloques reduciendo notablemente la resistencia a la compresión $\left[{ }^{2}\right]$.

El constante crecimiento demográfico mundial, que demanda la construcción de infraestructura de vivienda y social, debe conducir a la optimización de la producción de $\mathrm{CP}$ o a la búsqueda de materiales cementosos alternativos que resuelvan las necesidades de las generaciones presentes sin comprometer el bienestar de las generaciones futuras.

En este contexto surgen los materiales sustentables, que sustituyen parcial o totalmente al CP, formulados a partir de desechos urbanos e industriales, o con materias primas disponibles en la naturaleza sin recurrir a las altas temperaturas de clinquerización.

Este artículo presenta los resultados de una investigación sobre cementos compósitos base desechos inorgánicos: escoria de alto horno (EAH) y vidrio de desecho (VD). Ambos desechos carecen de un arreglo de largo alcance en la estructura atómica, lo que los hace termodinámicamente inestables y químicamente muy reactivos frente a los álcalis. La EAH es un subproducto de la obtención de arrabio que es la materia prima para la obtención de acero; químicamente está constituida principalmente de $\mathrm{CaO}, \mathrm{SiO}_{2}, \mathrm{Al}_{2} \mathrm{O}_{3}$ y $\mathrm{MgO}$. El VD proveniente de botellas de bebidas diversas, y es un vidrio soda-cal que se compone principalmente de $\mathrm{SiO}_{2}$, $\mathrm{Na}_{2} \mathrm{O}$ y $\mathrm{CaO}$ con pequeñas cantidades de aditivos usados para colorear o impartir ciertas propiedades específicas.
Partiendo de una estructura atómica amorfa, se dice que la EAH tiene propiedades hidráulicas latentes, pues al entrar en contacto con el agua ocurren reacciones de hidratación que forman nuevas fases con propiedades cementosas, sin embargo, la velocidad de reacción de la EAH con el agua es muy lenta debido a la formación de una capa ácida rica en $\mathrm{SiO}_{2}$ alrededor de los granos de EAH y actúa como una barrera que impide la penetración del agua hacia el interior de la estructura. Para aumentar su reactividad, existen diversas formas de activación de las reacciones. Una opción es la activación mecánica, que es el incremento del área superficial hasta 4000 ó $5000 \mathrm{~cm}^{2} / \mathrm{g}$; sin embargo, a valores muy altos se requiere más agua para obtener fluidez en los concretos, y en consecuencia la resistencia a la compresión se ve comprometida $\left[{ }^{3}\right]$. La activación química es otra opción, en la que se induce la disolución de la estructura amorfa mediante ataque con álcalis como $\mathrm{NaOH}$, silicato de sodio (SS), $\mathrm{Ca}(\mathrm{OH})_{2}, \mathrm{Na}_{2} \mathrm{SO}_{4}$ o combinaciones). Por último la activación térmica que induce la aceleración de la velocidad de las reacciones químicas, mediante el aumento de temperatura de curado.

En cementos $100 \%$ EAH los mejores resultados de resistencia a la compresión se han obtenido al utilizar bajas concentraciones de SS como agente activador y temperaturas de curado de $20^{\circ} \mathrm{C}\left[{ }^{4}\right]$. Por otro lado, la activación con $\mathrm{NaOH}$ y otros álcalis resulta en microestructuras porosas y menores propiedades mecánicas. Esto se debe a que el SS contribuye a la densificación microestructural mediante la condensación de un gel de sílice [4]. El curado a $20^{\circ} \mathrm{C}$ permite que un desarrollo gradual de las reacciones favoreciendo una buena consolidación microestructural, mientras que a $60^{\circ} \mathrm{C}$ (o a vapor) se alcanzan altas resistencias a edades tempranas, pero sin desarrollo más allá de los 28 días.

Por su composición química, el VD es una puzolana, similar a la ceniza volante (CV). En contraste con los cementos de $\mathrm{EAH}$, los cementos de $\mathrm{CV}$ requieren altas concentraciones alcalinas y altas temperaturas de curado para obtener buena resistencia a la compresión $\left[{ }^{5}\right]$.

El SS es el activador más efectivo en cementos de EAH o CV; sin embargo, es el más costoso, pues su producción implica la fusión de $\mathrm{Na}_{2} \mathrm{CO}_{3}$ y arena de $\mathrm{SiO}_{2}$ por $12-16$ horas a $1350-1450^{\circ} \mathrm{C}$. Por esta razón, en esta investigación el VD se sometió a una acción combinada de activación mecánica en un molino de bolas con activación química con una solución de $\mathrm{NaOH}$ a fin de solubilizar el $\mathrm{SiO}_{2}$ de la estructura amorfa. A este procedimiento se denominó activación mecanoquímica (AMQ). 


\section{METODOLOGÍA}

\section{Materiales}

Materiales cementantes: EAH y VD

La EAH se generó en Altos Hornos de México S. A. (AHMSA). El lote utilizado se molió en un molino de bolas (relación bolas/EAH de 10/1 en peso) por $2 \mathrm{~h}$ para obtener una finura de $4956 \mathrm{~cm}^{2} / \mathrm{g}$ medida por el método de Blaine. El vidrio consistió de botellas de diversas bebidas, $59 \%$ de vino, $27 \%$ de refresco y $14 \%$ de cerveza. Los contenedores de vidrio se trituraron y sometieron a molienda en iguales condiciones que la EAH hasta obtener una finura de $4100 \mathrm{~cm}^{2} / \mathrm{g}$.

La composición química de la EAH y del VD se determinó mediante fluorescencia de rayos $\mathrm{X}$. El VD presentó una composición química rica en $\mathrm{SiO}_{2}(70 \%)$ con aprox $13 \%$ de $\mathrm{Na}_{2} \mathrm{O}$ y de $\mathrm{CaO}$; la composición de la EAH presentó $33 \% \mathrm{SiO}_{2}, 38 \% \mathrm{CaO}, 11 \% \mathrm{Al}_{2} \mathrm{O}_{3}$ y $9 \%$ $\mathrm{MgO}$. En la Figura 1 se presenta el patrón de difracción de rayos X (DRX, Phillips, modelo PW3040) de la EAH y VD.

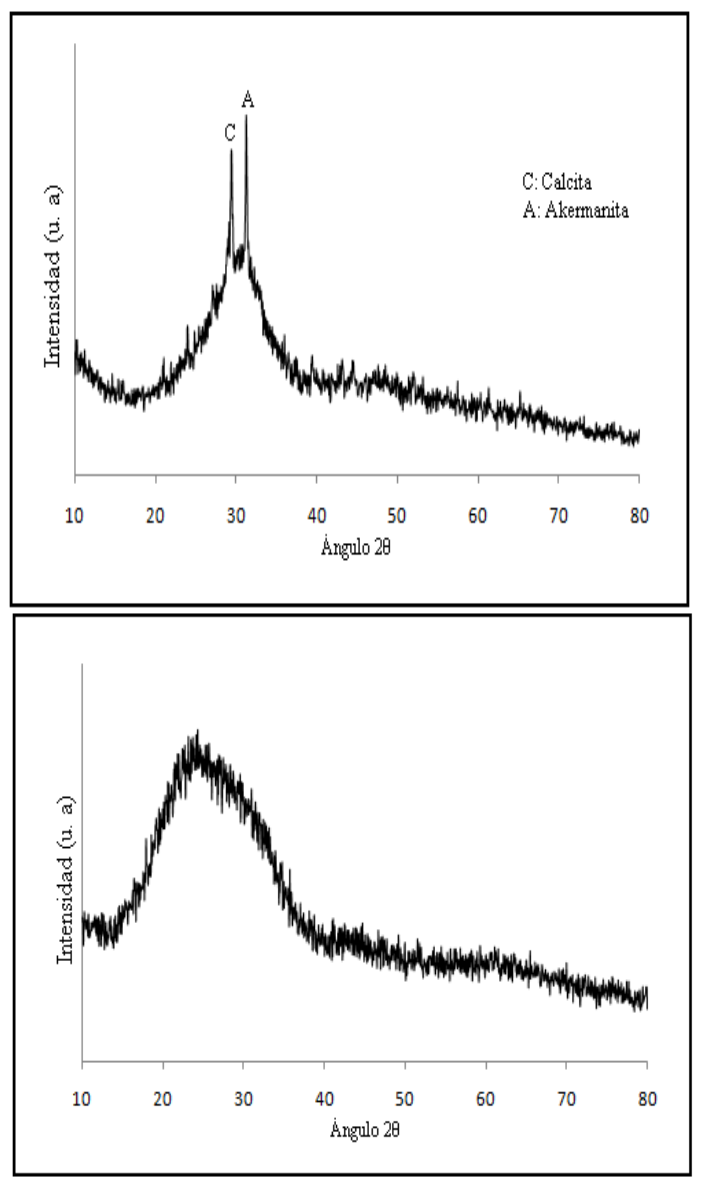

Figura 1. Patrón de DRX de la EAH (arriba) y VD (abajo)
Para la EAH se notó un halo amorfo entre $20-40^{\circ} 2 \theta$, que indica que es predominantemente amorfa, como resultado del rápido enfriamiento de la $\mathrm{EAH}$ a la salida del horno. Se observaron reflexiones de la fase akermanita, típica de escorias básicas, y de calcita $\left(\mathrm{CaCO}_{3}\right)$ que pudo originarse por la carbonatación del $\mathrm{CaO}$ al estar expuesta la EAH al ambiente. El patrón del VD mostró un halo amorfo entre $15-40^{\circ} 2 \theta$. El contraste entre ambos patrones indica que aunque ambas materias primas son amorfas, su composición química y arreglo estructural de corto alcance es diferente.

Soluciones activadoras: Los reactivos usados fueron $\mathrm{NaOH}$ y $\mathrm{Na}_{2} \mathrm{CO}_{3}$ grado industrial.

Arena de sílice: Para la elaboración de los morteros se utilizó arena de súlice 20-40 (arenas de sílice de Monterrey, S.A); ésta pasó la malla 20 (tamiz de $0.84 \mathrm{~mm}$ ) pero se retuvo en la malla 40 (tamiz de $0.42 \mathrm{~mm})$.

\section{Métodos}

Procedimiento de activación mecanoquímica:

La AMQ es la molienda mecánica de polvo de VD en un molino de bolas en presencia de una solución fuertemente alcalina $\left(\mathrm{NaOH}, \quad \mathrm{Na}_{2} \mathrm{CO}_{3} / \mathrm{NaOH}\right) . \quad \mathrm{La}$ solución alcalina ataca intensamente la red vítrea, adicionalmente, la disminución de tamaño de partícula inducida por la molienda favorece aun más la disolución del vidrio en el molino y en las reacciones de hidratación o geopolimerización posterior. Con esta activación combinada, se indujo una interfaz vidriosolución alcalina libre de reacción permanentemente, de manera que el ataque y la reducción de tamaño de partícula sean recurrentes en el tiempo. La combinación de todos estos factores en un solo proceso favorece la disolución de unidades estructurales $\mathrm{SiO}_{4}$ del VD hacia la solución, obteniéndose una suspensión de silicato de sodio.

Para la elaboración de todos los morteros, exceptuando aquellos $100 \% \mathrm{EAH}$, mediante AMQ se procesó una cantidad constante de VD $(25 \%$ del material cementante) en las soluciones alcalinas. Las variables estudiadas en la AMQ se describen en la Tabla 1 y se midió la cantidad de $\mathrm{SiO}_{2}$ y el $\mathrm{Ms}\left(\mathrm{SiO}_{2} / \mathrm{Na}_{2} \mathrm{O}\right)$ de las soluciones resultantes. 
Espinoza L. y Escalante J.

Tabla 1. Variables y niveles investigados para AMQ

\begin{tabular}{|c|c|c|c|c|}
\hline $\begin{array}{c}\text { Tiempo de } \\
\text { molienda } \\
\text { (Horas) }\end{array}$ & $\begin{array}{c}\text { Rel. p/p } \\
\text { Bolas/VD }\end{array}$ & $\begin{array}{c}\text { Rel. p/p } \\
\text { Agua/Cemento }\end{array}$ & $\begin{array}{c}\text { Rel. p/p } \\
\mathrm{Na}_{2} \mathrm{CO}_{3} / \mathrm{NaOH}\end{array}$ & $\% \mathrm{Na}_{2} \mathrm{O}$ \\
\hline 0.5 & $5: 1$ & 0.30 & $0: 1$ & 4 \\
\hline 1.5 & $10: 1$ & 0.35 & $1: 1$ & 8 \\
\hline 3 & $15: 1$ & 0.40 & $2: 1$ & 12 \\
\hline
\end{tabular}

Tabla 2. Arreglo ortogonal $\mathrm{L}_{9}\left(3^{5}\right)$ utilizado en la Investigación

\begin{tabular}{|c|c|c|c|c|c|}
\hline 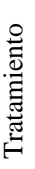 & 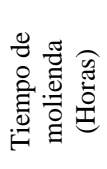 & 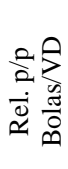 & 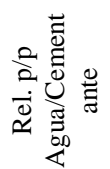 & 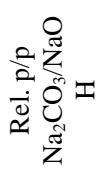 & $\begin{array}{l}0 \\
Z_{0} \\
0\end{array}$ \\
\hline 1 & 0.5 & $5: 1$ & 0.30 & $0: 1$ & 4 \\
\hline 2 & 0.5 & $5: 1$ & 0.40 & 2:1 & 8 \\
\hline 3 & 0.5 & $15: 1$ & 0.30 & 2:1 & 12 \\
\hline 4 & 1.5 & 10:1 & 0.40 & $0: 1$ & 12 \\
\hline 5 & 1.5 & $15: 1$ & 0.35 & $0: 1$ & 8 \\
\hline 6 & 1.5 & $15: 1$ & 0.40 & 1:1 & 4 \\
\hline 7 & 3 & $5: 1$ & 0.35 & $1: 1$ & 12 \\
\hline 8 & 3 & $10: 1$ & 0.30 & $1: 1$ & 8 \\
\hline 9 & 3 & $10: 1$ & 0.35 & $2: 1$ & 4 \\
\hline
\end{tabular}

Para el estudio de estas variables, un experimento factorial $3^{5}$ permite investigar todas las combinaciones de variables y niveles posibles. La mejor forma de identificar la combinación óptima de niveles para obtener el mayor Ms sería realizar los 243 experimentos, pero esto implica altos costos y tiempos muy largos. Como alternativa se utilizó el método Taguchi, que se basa en la utilización de arreglos ortogonales. Estos indican cuáles y cuántos experimentos se deben realizar para un número de variables y de niveles determinado. Así, para cinco variables a tres niveles cada una, el arreglo ortogonal que debe utilizarse es un $\mathrm{L}_{9}\left(3^{5}\right)$ y se muestra en la

Tabla $2\left[{ }^{6}\right]$.

Después de realizar cada tratamiento se obtuvo una suspensión compuesta de vidrio disuelto, solución alcalina y vidrio sin disolver. Se tomó una muestra representativa de cada suspensión obtenida, se centrifugó a 3,000 rpm por 10 minutos para separar el vidrio sin disolver de la solución de silicato de sodio (SS) obtenida. La solución de SS se envió a análisis por espectrometría de emisión atómica por ICP. Para cada tratamiento se tomaron 3 lecturas de la cantidad de $\mathrm{Na}_{2} \mathrm{O}(\mathrm{g} / \mathrm{l})$ y de $\mathrm{SiO}_{2}(\mathrm{~g} / \mathrm{l})$.

Preparación de morteros con suspensiones obtenidas mediante $A M Q$ :

Mediante el análisis de Taguchi se seleccionaron las condiciones óptimas que permiten obtener los mayores valores de $\mathrm{Na}_{2} \mathrm{O}$ y $\mathrm{SiO}_{2}$ en solución. Se evaluó el efecto del $\% \mathrm{Na}_{2} \mathrm{O}$ (a dos niveles) de las suspensiones resultantes sobre las propiedades mecánicas, microestructura y compuestos de hidratación de cementos compósitos EAH-VD (sustitución con 0, 25, 75 y $100 \%$ VD).

Para preparar los morteros se homogenizaron los polvos de EAH y VD por 5 minutos en una mezcladora de paletas según el compósito a fabricar, se adicionó la suspensión alcalina correspondiente preparada bajo las condiciones óptimas, y se mezcló por 10 minutos más. Una fracción de la pasta se vació en moldes cúbicos para caracterizar por DRX y el restante se mezcló con arena de sílice 20-40 en relación 3:1 en peso y se mezcló por 10 minutos más. Los morteros se vaciaron en barras de $4 \times 4 \times 48 \mathrm{~cm}$ y se vibraron por 90 segundos para luego curarse a 20 ó $60^{\circ} \mathrm{C}$ según correspondía hasta por 28 días. Se midió la Resistencia Mecánica a la Compresión (RMC) de cada compósito (en cubos de $4 \mathrm{cms}$ por lado) a $\operatorname{los} 1,7,14$ y 28 días. Se caracterizaron muestras selectas a 28 días por Microscopía Electrónica de Barrido (MEB-EDS) y Difracción de Rayos X (DRX).

\section{RESULTADOS Y DISCUSIÓN}

\section{Activación mecanoquímica}

La Tabla 3 presenta los valores promedio de la cantidad de $\mathrm{SiO}_{2}, \mathrm{Na}_{2} \mathrm{O}$ y $\mathrm{Ms}\left(\mathrm{SiO}_{2} / \mathrm{Na}_{2} \mathrm{O}\right)$ obtenido para los 9 tratamientos.

Tabla 3. Valores promedio de la cantidad de $\mathrm{SiO}_{2}, \mathrm{Na}_{2} \mathrm{O}$ y Ms obtenido para cada tratamiento

\begin{tabular}{|c|c|c|c|c|c|c|c|c|}
\hline 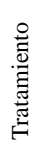 & 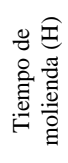 & 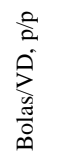 & 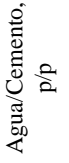 & 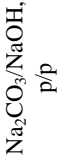 & $\begin{array}{l}0 \\
\text { Zू } \\
\text { Zू }\end{array}$ & 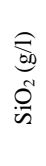 & 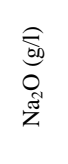 & $\sum^{n}$ \\
\hline 1 & 0.5 & $5: 1$ & 0.30 & $0: 1$ & 4 & 1.8 & 156.7 & 0.012 \\
\hline 2 & 0.5 & $5: 1$ & 0.40 & 2:1 & 8 & 2.5 & 196.4 & 0.013 \\
\hline 3 & 0.5 & 15:1 & 0.30 & $2: 1$ & 12 & 3.8 & 244.4 & 0.016 \\
\hline 4 & 1.5 & 10:1 & 0.40 & $0: 1$ & 12 & 8.4 & 295.0 & 0.029 \\
\hline
\end{tabular}


Espinoza L. y Escalante J.

\begin{tabular}{|c|c|c|c|c|c|c|c|c|}
\hline 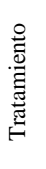 & 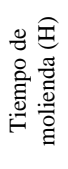 & 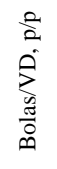 & 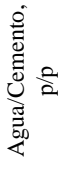 & 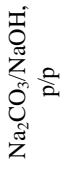 & 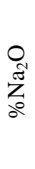 & 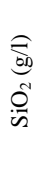 & 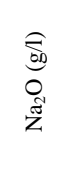 & $\sum^{\infty}$ \\
\hline 5 & 1.5 & $15: 1$ & 0.35 & $0: 1$ & 8 & 6.9 & 233.4 & 0.030 \\
\hline 6 & 1.5 & $15: 1$ & 0.40 & $1: 1$ & 4 & 3.3 & 97.4 & 0.034 \\
\hline 7 & 3 & $5: 1$ & 0.35 & $1: 1$ & 12 & 4.9 & 245.5 & 0.020 \\
\hline 8 & 3 & 10:1 & 0.30 & $1: 1$ & 8 & 5.8 & 188.6 & 0.031 \\
\hline 9 & 3 & 10:1 & 0.35 & $2: 1$ & 4 & 4.5 & 96.5 & 0.047 \\
\hline
\end{tabular}

Se observó que los valores de Ms resultaron más bajos que los comúnmente reportados para activación química de la EAH, idealmente de Ms entre 1-1.5 para favorecer la RMC [5]. Aún con los resultados obtenidos, se continuó con la experimentación. Los resultados se muestran en la Figura 2 y Figura 3, de las cuales es posible obtener el valor óptimo de cada una de las variables involucradas, los cuales se presentan en la Tabla 4.

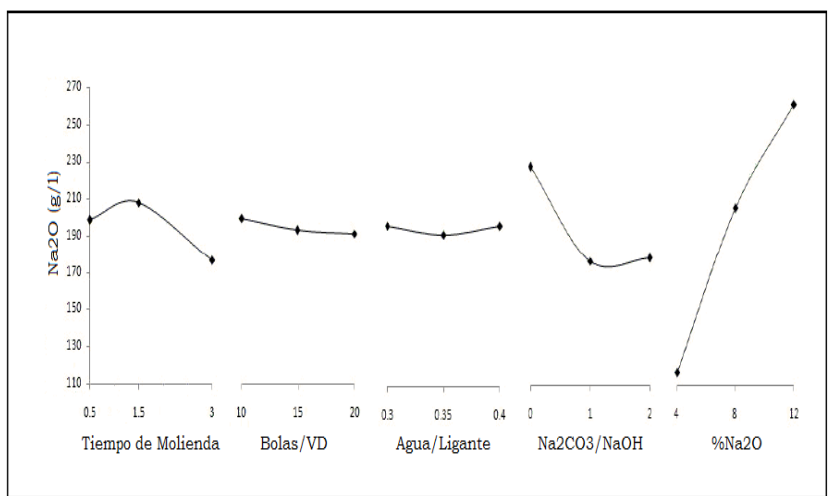

Figura 2. Efecto de las variables de la AMQ sobre el contenido de $\mathrm{Na}_{2} \mathrm{O}(\mathrm{g} / \mathrm{l})$ promedio.

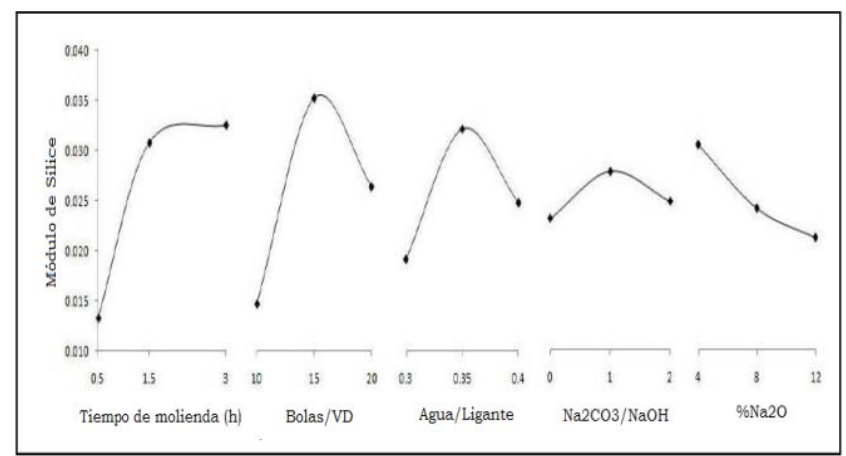

Figura 3. Efecto de las variables de la AMQ sobre el Ms promedio.

Según el método Taguchi, una variable tendrá mayor influencia sobre el proceso al ser más vertical y menor influencia al ser más horizontal $\left[{ }^{7},{ }^{89},\right]$. Así, puede decirse que las variables de mayor influencia para obtener altos contenidos de $\mathrm{Na}_{2} \mathrm{O}$ en solución (y altos contenidos de $\mathrm{SiO}_{2}$ disuelta) son el $\% \mathrm{Na}_{2} \mathrm{O}$, la relación $\mathrm{Na}_{2} \mathrm{CO}_{3} / \mathrm{NaOH}$ y el tiempo de molienda; y que la relación bolas/vidrio y la relación agua/ligante tuvieron poca influencia. El predominio de los iones $\mathrm{OH}^{-}$durante la AMQ provocó la destrucción de la red vítrea por la ruptura de los enlaces $\mathrm{Si}-\mathrm{O}-\mathrm{Si}$ y $\mathrm{Si}-\mathrm{O}-\mathrm{Na}$ del VD causando que las unidades estructurales de $\mathrm{SiO}_{4}$ y Na pasaran a solución. La presencia de $\mathrm{CO}_{3}{ }^{2-}$ en el activador alcalino redunda en una menor concentración de iones $\mathrm{OH}^{-}$, acidificando el sistema y disminuyendo la intensidad del ataque sobre el retículo vítreo $\left[{ }^{10}\right]$. Por ello, al aumentar la relación en peso $\mathrm{Na}_{2} \mathrm{CO}_{3} / \mathrm{NaOH}$ de $0: 1$ a $1: 1$ y $2: 1$, disminuyó la cantidad de $\mathrm{Na}_{2} \mathrm{O}(\mathrm{g} / \mathrm{l})$ en solución. De igual manera, al disminuir el $\% \mathrm{Na}_{2} \mathrm{O}$ se disminuyó la concentración de iones $\mathrm{OH}^{-}$con la consiguiente disminución de la intensidad del ataque sobre la estructura vítrea.

Tabla 4. Valores óptimos de cada variable según el análisis con el Método Taguchi

\begin{tabular}{|c|c|c|c|c|c|}
\hline & \multicolumn{5}{|c|}{ Condiciones óptimas } \\
\hline 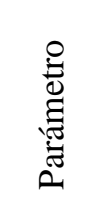 & 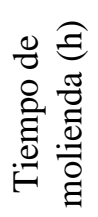 & $\begin{array}{l}\sum_{\substack{0 \\
0 \\
0 \\
0}} \\
\infty\end{array}$ & 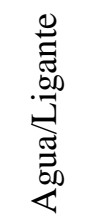 & 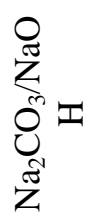 & $\begin{array}{l}0 \\
Z^{\circ} \\
\text { Zू }\end{array}$ \\
\hline $\mathrm{Na}_{2} \mathrm{O}$ & 1.5 & $10 / 1$ & 0.30 & $0: 1$ & 12 \\
\hline Ms & 3 & $15 / 1$ & 0.35 & $1: 1$ & 4 \\
\hline
\end{tabular}

Resistencia mecánica a la compresión de morteros con $A M Q$

De acuerdo al análisis Taguchi presentado previamente en la Figura 2 y Figura 3 se obtuvieron los valores óptimos de cada variable de procesamiento de la AMQ que permiten obtener soluciones con los más altos valores de $\% \mathrm{Na}_{2} \mathrm{O}$ y $\mathrm{Ms}$ (ver Tabla 4). Se tomaron $500 \mathrm{~g}$ de VD y se procesaron por AMQ bajo las condiciones descritas en la

Tabla 2. Las suspensiones resultantes de la AMQ se utilizaron para activar morteros de cementos compósitos de EAH con sustituciones de $25 \%, 75 \%$ y $100 \%$ de VD y se curaron a $20^{\circ} \mathrm{C}$ ó $60^{\circ} \mathrm{C}$ hasta por 28 días. Adicionalmente como referencia se prepararon morteros $100 \%$ EAH con activación alcalina convencional. La activación alcalina convencional se refiere a aquella activación química que se lleva a cabo con soluciones de 
Espinoza L. y Escalante J.

$\mathrm{NaOH}$ o combinación de $\mathrm{Na}_{2} \mathrm{CO}_{3} / \mathrm{NaOH}$. Los resultados se muestran en la Tabla 5 , cuya nomenclatura usada es la siguiente: $w-x-y-z$, donde: $w$ es $\%$ en peso de EAH en el compósito, $x$ es $\%$ de VD, $y$ es $\% \mathrm{Na}_{2} \mathrm{O}$ y $z$ es la temperatura de curado.

La Figura 4 presenta los valores más altos de RMC a 28 días de morteros con AMQ. Los morteros con $100 \%$ EAH alcanzaron la mayor RMC con $4 \% \mathrm{Na}_{2} \mathrm{O}$ y curado a $20^{\circ} \mathrm{C}$, el incremento de la concentración de $\mathrm{Na}_{2} \mathrm{O}$ a $12 \%$ resultó contraproducente debido a un exceso de $\mathrm{Na}_{2} \mathrm{O}$ que ocasiona reacciones de carbonatación, disminuyendo la RMC. Estos resultados coinciden con la literatura científica para pastas $100 \% \mathrm{EAH}$ activadas con $\mathrm{NaOH}\left[5,{ }^{11},{ }^{12}\right]$. En contraste, los morteros con $100 \%$ VD alcanzaron su mayor RMC con $12 \% \mathrm{Na}_{2} \mathrm{O}$ y curado a $60^{\circ} \mathrm{C}$. La EAH y VD requirieron condiciones de activación química y térmica diferentes, y puede deducirse que el VD es menos reactivo que la EAH, pues el VD requiere de condiciones muy agresivas para provocar la disolución de su estructura vítrea; sin embargo, con VD se alcanzaron hasta 48MPa mientras que para EAH se alcanzaron 26.6 MPa a 28 días.

Tabla 5. RMC de morteros con AMQ

\begin{tabular}{|c|c|c|c|c|c|c|c|c|}
\hline \multirow[t]{2}{*}{ Sistema } & \multirow{2}{*}{ 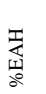 } & \multirow{2}{*}{$\sum_{0}^{\circ}$} & \multirow{2}{*}{$\begin{array}{l}0 \\
Z_{\tilde{J}} \\
Z_{0}\end{array}$} & \multirow{2}{*}{ 苞。 } & \multicolumn{4}{|c|}{ Tiempo de curado (días) } \\
\hline & & & & & 1 & 7 & 14 & 28 \\
\hline $100-0-4-20^{*}$ & 100 & 0 & 4 & 20 & $\begin{array}{c}20.4 \\
(3.54)\end{array}$ & $\begin{array}{c}20.7 \\
(1.71)\end{array}$ & $\begin{array}{c}22.8 \\
(1.29)\end{array}$ & $\begin{array}{c}26.6 \\
(2.97)\end{array}$ \\
\hline $75-25-4-20$ & 75 & 25 & & & $\begin{array}{c}15.4 \\
(1.53)\end{array}$ & $\begin{array}{c}21.2 \\
(2.01)\end{array}$ & $\begin{array}{c}15.9 \\
(0.42)\end{array}$ & $\begin{array}{c}13.6 \\
(1.10)\end{array}$ \\
\hline $25-75-4-20$ & 25 & 75 & & & $\begin{array}{c}7.9 \\
(0.63)\end{array}$ & $\begin{array}{c}9.3 \\
(1.08)\end{array}$ & $\begin{array}{c}9.3 \\
(2.06)\end{array}$ & $\begin{array}{c}9.4 \\
(1.48)\end{array}$ \\
\hline $0-100-4-20$ & 0 & 100 & & & $\begin{array}{c}7.1 \\
(0.26)\end{array}$ & $\begin{array}{c}14.5 \\
(0.67)\end{array}$ & $\begin{array}{c}14.9 \\
(1.05)\end{array}$ & $\begin{array}{c}16.1 \\
(0.32)\end{array}$ \\
\hline $100-0-4-60 *$ & 100 & 0 & & 60 & $\begin{array}{c}20.9 \\
(5.76)\end{array}$ & $\begin{array}{c}14.8 \\
(0.97)\end{array}$ & $\begin{array}{c}20.7 \\
(3.91)\end{array}$ & $\begin{array}{c}21.0 \\
(3.40)\end{array}$ \\
\hline $75-25-4-60$ & 75 & 25 & & & $\begin{array}{c}17.1 \\
(3.73)\end{array}$ & $\begin{array}{c}19.3 \\
(2.97)\end{array}$ & $\begin{array}{c}12.1 \\
(2.89)\end{array}$ & $\begin{array}{c}8.3 \\
(0.47)\end{array}$ \\
\hline $25-75-4-60$ & 25 & 75 & & & $\begin{array}{c}13.2 \\
(3.94)\end{array}$ & $\begin{array}{c}16.7 \\
(1.44)\end{array}$ & $\begin{array}{c}10.3 \\
(1.01)\end{array}$ & $\begin{array}{c}10.7 \\
(0.57)\end{array}$ \\
\hline $0-100-4-60$ & 0 & 100 & & & $\begin{array}{c}16.2 \\
(3.70)\end{array}$ & $\begin{array}{c}21.1 \\
(2.09)\end{array}$ & $\begin{array}{c}15.2 \\
(0.79)\end{array}$ & $\begin{array}{c}16.7 \\
(1.12)\end{array}$ \\
\hline $100-0-12-20^{*}$ & 100 & 0 & 12 & 20 & $\begin{array}{c}7.3 \\
(1.70)\end{array}$ & $\begin{array}{c}11.5 \\
(1.66)\end{array}$ & $\begin{array}{c}10.2 \\
(1.52)\end{array}$ & $\begin{array}{c}10.9 \\
(1.65)\end{array}$ \\
\hline $75-25-12-20$ & 75 & 25 & & & $\begin{array}{c}9.7 \\
(1.93)\end{array}$ & $\begin{array}{c}6.3 \\
(1.52)\end{array}$ & $\begin{array}{c}8.6 \\
(0.70)\end{array}$ & $\begin{array}{c}12.3 \\
(1.27)\end{array}$ \\
\hline $25-75-12-20$ & 25 & 75 & & & $\begin{array}{c}3.1 \\
(0.99)\end{array}$ & $\begin{array}{c}4.0 \\
(0.19)\end{array}$ & $\begin{array}{c}4.6 \\
(0.58)\end{array}$ & $\begin{array}{c}7.2 \\
(0.37)\end{array}$ \\
\hline $0-100-12-20$ & 0 & 100 & & & $\begin{array}{c}1.1 \\
(0.17)\end{array}$ & $\begin{array}{c}1.4 \\
(0.01\end{array}$ & $\begin{array}{c}1.7 \\
(0.14)\end{array}$ & $\begin{array}{c}2.4 \\
(0.14)\end{array}$ \\
\hline $100-0-12-60^{*}$ & 100 & 0 & & 60 & $\begin{array}{c}4.5 \\
(1.69)\end{array}$ & $\begin{array}{c}8.1 \\
(1.75)\end{array}$ & $\begin{array}{c}8.0 \\
(0.33)\end{array}$ & $\begin{array}{c}8.4 \\
(0.40)\end{array}$ \\
\hline $75-25-12-60$ & 75 & 25 & & & $\begin{array}{c}10.5 \\
(1.25)\end{array}$ & $\begin{array}{c}7.7 \\
(0.19)\end{array}$ & $\begin{array}{c}8.8 \\
(0.74)\end{array}$ & $\begin{array}{c}17.7 \\
(0.82)\end{array}$ \\
\hline $25-75-12-60$ & 25 & 75 & & & $\begin{array}{c}7.3 \\
(1.71)\end{array}$ & $\begin{array}{c}23.6 \\
(2.32)\end{array}$ & $\begin{array}{c}37.6 \\
(2.74)\end{array}$ & $\begin{array}{r}32.3 \\
(4.82)\end{array}$ \\
\hline $0-100-12-60$ & 0 & 100 & & & $\begin{array}{c}4.2 \\
(0.58)\end{array}$ & $\begin{array}{c}44.4 \\
(6.18)\end{array}$ & $\begin{array}{c}43.5 \\
(3.76)\end{array}$ & $\begin{array}{c}48.0 \\
(4.58)\end{array}$ \\
\hline
\end{tabular}

*Morteros con activación alcalina tradicional; Los valores entre paréntesis corresponden a la desviación estándar para un promedio de tres muestras.
Era de esperarse que para el compósito 75-25-y-z las condiciones de activación que reportaran mayor RMC fueran $4 \% \mathrm{Na} 2 \mathrm{O}$ y $20^{\circ} \mathrm{C}$, dado el alto contenido de $\mathrm{EAH}$; sin embargo, los resultados indicaron que se requieren condiciones de activación similares a las del cemento 100\%VD. Para el compósito 50-50-y-z las condiciones de activación requeridas fueron similares a las del cemento 100-0-4-20. Finalmente, para el compósito 25$75-y-z$ las condiciones de activación requeridas fueron similares a las del cemento 0-100-12-60, por encontrarse el VD en mayor porcentaje.

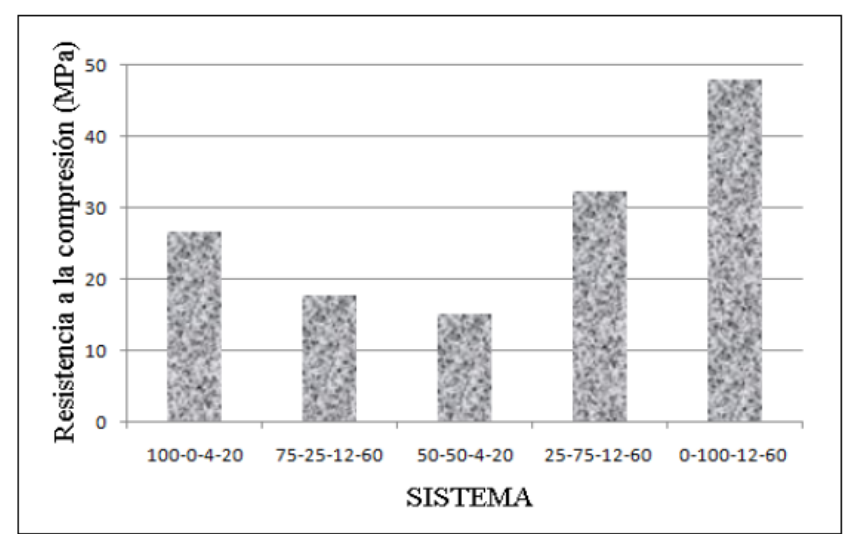

Figura 4. Mayores valores de RMC de morteros con AMQ curados a 28 días

En la Figura 5 se presenta el efecto de la temperatura de curado sobre la RMC de morteros de cemento 100-0-yz, 0-100-y-z, 75-25-y-z y 25-75-y-z a los 28 días de curado. Para cementos 100-0-4-z y 100-0-12-z, el aumento de la temperatura de 20 a $60^{\circ} \mathrm{C}$ redujo la $\mathrm{RMC}$, esto es acorde con reportes de EAH activada con $\mathrm{NaOH}$ [4]. Por otro lado para cementos 0-100-12-z, el incremento de la temperatura de curado incrementó la RMC; para cementos $0-100-4-z$, se observaron pocas diferencias en la RMC durante el curado a 20 ó $60^{\circ} \mathrm{C}$.

Para cementos compósitos 75-25-12-z y 25-75-12-z, la RMC mejoró con el curado a $60^{\circ} \mathrm{C}$. El compósito 75-254-z mostró la mayor RMC con el curado a $20^{\circ} \mathrm{C}$, por el contrario, el compósito 25-75-4-z mostró mejor RMC con el curado a $60^{\circ} \mathrm{C}$. De manera general, se observó incremento de RMC al incrementar la temperatura solo para $12 \% \mathrm{Na}_{2} \mathrm{O}$ y en presencia de $\mathrm{VD}$; mientras que para $4 \% \mathrm{Na}_{2} \mathrm{O}$ el incremento de la temperatura no fue favorable en caso alguno. 
Espinoza L. y Escalante J.

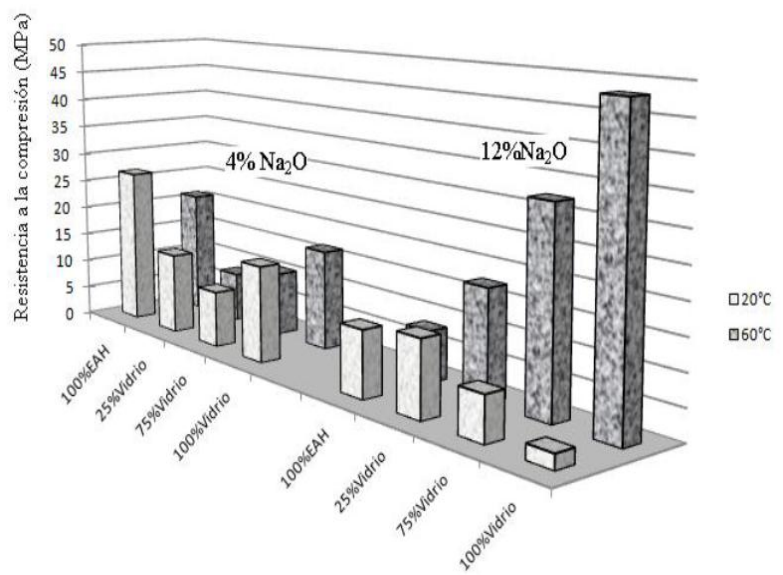

Figura 5. Efecto de la temperatura de curado sobre la RMC de morteros 100-0-y-z, 0-100-y-z, 75-25-y-z y 25-75-y-z

La Figura 6 presenta el efecto del $\% \mathrm{Na}_{2} \mathrm{O}$ sobre la RMC a 28 días de curado para diversos morteros. Para cementos 100-0-y-20 y 100-0-y-60, el incremento de $\mathrm{Na}_{2} \mathrm{O}$ de $4 \%$ a $12 \%$ resultó en una disminución de la RMC. Esta tendencia ya ha sido reportada en la literatura $[5,11,12]$ y se atribuyó al exceso de $\mathrm{Na}_{2} \mathrm{O}$ que no participó en las reacciones de formación de productos de reacción y a la consecuente formación de $\mathrm{Na}_{2} \mathrm{CO}_{3}$. Por otra parte, Pahir $\left[{ }^{13}\right]$ expuso que la disminución de RMC se debió a las altas concentraciones de iones $\mathrm{OH}^{-}$. Las altas concentraciones de iones $\mathrm{OH}^{-}$en la solución activadora limitan la solubilidad del calcio, pues el $\mathrm{Ca}^{2+}$ que logra solubilizarse de la estructura vítrea de la $\mathrm{EAH}$ reacciona con los iones $\mathrm{OH}^{-}$en exceso, posiblemente formando una capa de $\mathrm{Ca}(\mathrm{OH})_{2}$ sobre los granos de EAH y que dificulta la salida de más iones $\mathrm{Ca}^{2+}$ necesarios para formar productos de reacción como el $\mathrm{C}-\mathrm{S}-\mathrm{H}\left[{ }^{14}\right]$. El $\mathrm{Ca}(\mathrm{OH})_{2}$ es inestable y tiende a formar $\mathrm{CaCO}_{3}$ reacción con el $\mathrm{CO}_{2}$ de la atmósfera, en detrimento de la RMC. En esta investigación no se encontró evidencia de que haya ocurrido lo expuesto por Pahir.

Para cementos 0-100-y-60, la mayor RMC fue por activación con $12 \% \mathrm{Na}_{2} \mathrm{O}$, un reporte de cementos $100 \% \mathrm{MK}[12]$, indicó que el uso de bajos $\% \mathrm{Na}_{2} \mathrm{O}$ $(<15 \%)$ fue insuficiente para provocar la disolución del MK y generar la condensación de nuevos productos ligantes que favorecen la RMC mediante la consolidación de la matriz de productos de reacción. Los cementos compósitos 75-25-y-60 y 25-75-y-60 también aumentaron su RMC al activarse con un mayor contenido de $\mathrm{Na}_{2} \mathrm{O}$.

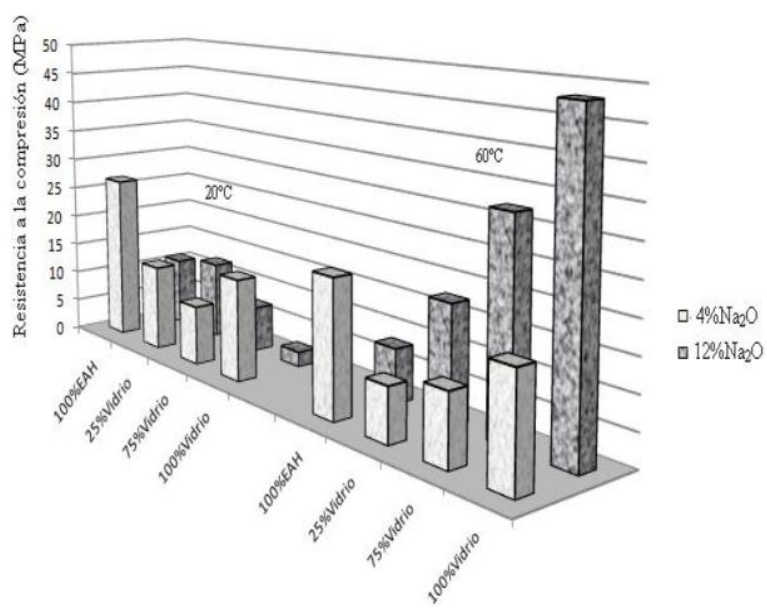

Figura 6. Efecto del $\% \mathrm{Na}_{2} \mathrm{O}$ sobre la $\mathrm{RMC}$ de morteros $100-0-y-z, 0-100-y-z, 75-25-y-z$ y $25-75-y-$ $\mathrm{Z}$.

Los cementos 0-100-y-20 mostraron una disminución en la $\mathrm{RMC}$ al aumentar el $\% \mathrm{Na}_{2} \mathrm{O}$ de 4 a $12 \%$. Las altas temperaturas favorecen el proceso de corrosión del VD en ambientes alcalinos, por ello durante el curado a $20^{\circ} \mathrm{C}$ no todo el $\mathrm{Na}_{2} \mathrm{O}$ alcanzó a reaccionar con el VD, quedando $\mathrm{Na}_{2} \mathrm{O}$ remanente que reaccionó con el $\mathrm{CO}_{2}$ atmosférico disminuyendo la RMC. Para el mortero compósito 25-75-y-20 también se observó la reducción de la RMC al aumentar el $\% \mathrm{Na}_{2} \mathrm{O}$. Sobre la superficie del sistema 0-100-12-20 apareció eflorescencia, la cual se raspó y analizó mediante DRX, determinándose que se trataba de termonatrita, $\mathrm{Na}_{2} \mathrm{CO}_{3} \cdot \mathrm{H}_{2} \mathrm{O}$.

\section{Difracción de Rayos $X, D R X$}

La Figura 7 presenta el patrón de DRX de la EAH sin reacción y de los cementos 100-0-12-60 y 75-25-12-60. Se observó una reflexión definida a $30^{\circ} 2 \theta$ correspondiente al gel C-S-H que se traslapa con la de la calcita; ésta fue más intensa para el cemento 100-0-1260 debido al mayor \%EAH, o bien a la mayor cristalinidad del C-S-H formado. En ambos cementos se observó la presencia de una fase tipo hidrotalcita a $11^{\circ}$, $23^{\circ}$ y $35^{\circ} 2 \theta$. Para el compósito 75-25-12-60 se observaron reflexiones de una fase minoritaria de zeolita carbonatada tipo cancrinita $\left[\mathrm{Na}_{6} \mathrm{CaAl}_{6} \mathrm{Si}_{6}\left(\mathrm{CO}_{3}\right) \mathrm{O}_{24} \cdot 2 \mathrm{H}_{2} \mathrm{O}\right]$. Generalmente las zeolitas se sintetizan por conversión alcalina de $\mathrm{CV}$ (materiales silíceos o silicoaluminosos), mediante la disolución de la sílice y/o alúmina, con la posterior precipitación de la zeolita. Algunos reportes [15] han demostrado que las mejores condiciones de síntesis de la cancrinita son de altas concentraciones de activador (3M y $5 \mathrm{M}$ de $\mathrm{NaOH}$ ) y altas temperaturas; así, es posible que la cancrinita se haya formado por la interacción del VD 
y EAH con la solución de $\mathrm{NaOH}$ y a $60^{\circ} \mathrm{C}$. A medida que aumenta la cantidad de $\mathrm{SiO}_{2}$ en el medio se requiere más tiempo para que cristalicen las zeolitas $\left[{ }^{15}\right]$, por lo cual no se espera encontrar cancrinita en compósitos con $50 \%, 75 \%$ y $100 \%$ VD activados con $12 \% \mathrm{Na}_{2} \mathrm{O}$ y curados a $60^{\circ} \mathrm{C}$. Las zeolitas se consideran productos secundarios que en exceso pueden debilitar la estructura $\left[{ }^{16}\right]$ reduciendo la RMC.

El halo amorfo del cemento 100-0-12-60 disminuyó comparado con el halo de la EAH sin reacción. También el halo amorfo del compósito 75-25-12-60 se extendió a ángulos bajos por la presencia de VD y disminuyó debido a la disolución de la estructura vítrea. La diferencia en los halos entre ambos cementos indica que el producto de reacción aunque amorfo, posiblemente es de distinto arreglo estructural de corto alcance $\mathrm{y}$ diferentes propiedades mecánicas.

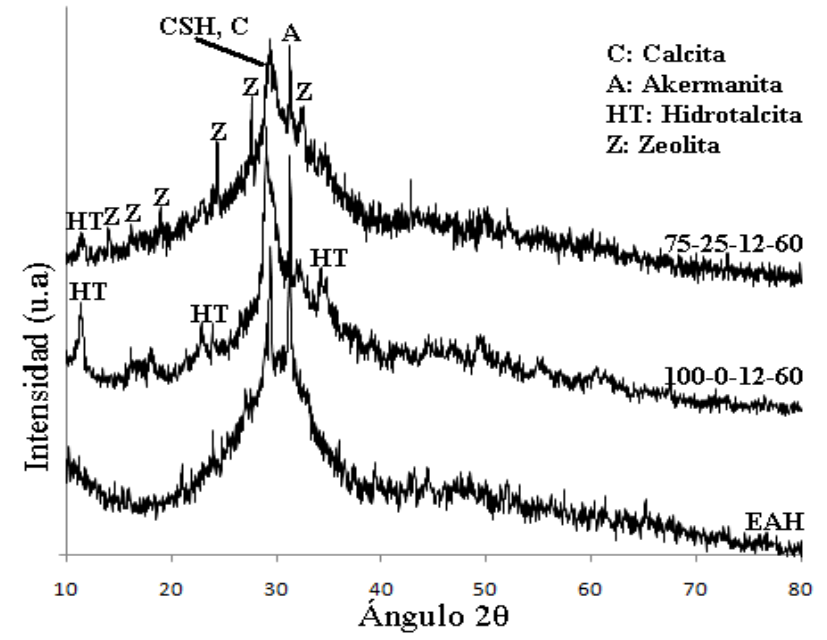

Figura 7. Patrón de DRX de la EAH sin reaccionar y de cementos 100-0-12-60 y 75-25-12-60

La Figura 8 presenta el patrón DRX del VD sin reaccionar y de cementos 0-100-4- 20 y 0-100-4-60. Se ha reportado $\left[16,{ }^{17}\right]$ que en el MK el halo amorfo aparece a $20-30^{\circ} 2 \theta$ y posterior a la activación alcalina el halo se desplaza hacia valores de $25-35^{\circ} 2 \theta$; esto sugiere la formación de productos de reacción de un polímero inorgánico alcalino de estructura amorfa diferente al material de partida. Este desplazamiento del halo amorfo no se observó para ambos cementos base VD, aunque si se observó un cambio en la forma del halo amorfo que inicialmente mostraba asimetría con mayor intensidad a ángulos bajos y luego de la AMQ se apreció más simétrico. Dicho cambio podría considerarse un indicador de la reacción del VD formando nuevos productos de carácter cementoso, con estructura amorfa de composición química diferente y con ganancia de resistencia mecánica a 28 días de curado.

La Figura 9 presenta el patrón de DRX del VD sin reaccionar y de cementos 0- 100-4-60 y 0-100-12-60. Similar a la Figura 8, para ambos cementos no se observó desplazamiento del halo amorfo después de la AMQ, pero si un cambio en la forma del halo que inicialmente mostraba asimetría con mayor intensidad a ángulos bajos y posterior a la AMQ se apreció más simétrico. Para el cemento 0-100-12-60 (la mayor RMC de todos los cementos elaborados $48 \mathrm{MPa}$ a 28 días), se observó una reflexión bien definida a $30^{\circ} 2 \theta$. La indexación de una sola reflexión resulta difícil, sin embargo, la literatura indica $\left[{ }^{18}\right]$ que ésta y otras reflexiones de menor intensidad que pueden presentarse $\mathrm{a} \approx 50^{\circ}$ y $55^{\circ} 2 \theta$ indican la presencia de C-S-H pobremente cristalino. Una alternativa razonable es la presencia de $\mathrm{CaCO}_{3}$, que se traslapa con la del C-S-H a $30^{\circ} 2 \theta$. Sin embargo, dado que en la literatura [18] el pico a $30^{\circ} 2 \theta$ es repetidamente identificado y confirmado con técnicas complementarias (espectroscopía por infrarrojo) como C-S-H, se descarta la presencia de $\mathrm{CaCO}_{3}$.

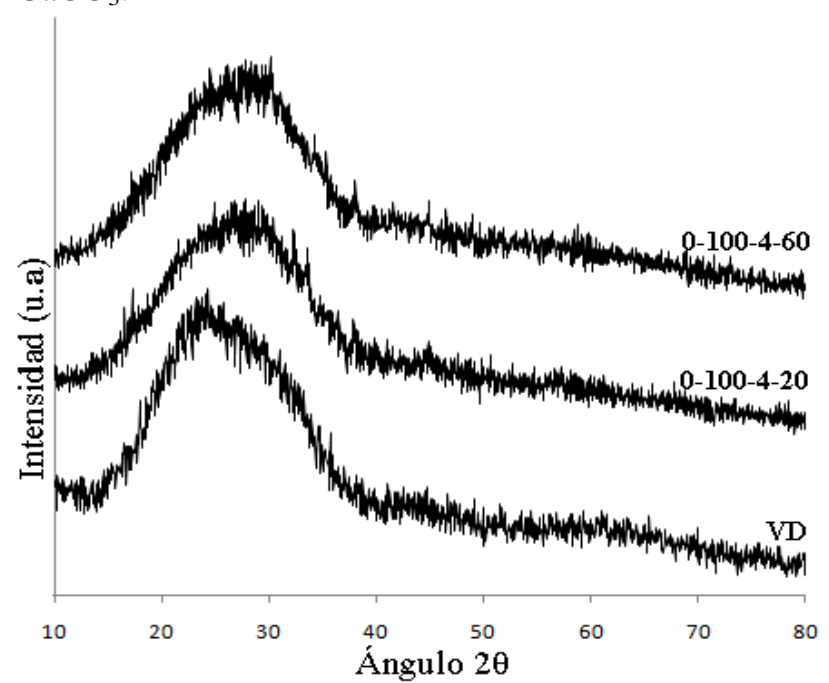

Figura 8. Patrón de DRX del VD sin reaccionar y de cementos 0-100-4-20 y 0-100-4-60

\section{Microscopía electrónica de barrido (MEB)}

Todas las muestras analizadas fueron aquellas con 28 días de curado. La Figura 10 A muestra la microestructura del mortero 100-0-4-60 (21 MPa), la parte B presenta la microestructura del mortero 50-50-460 (14.9 MPa). La RMC del cemento 50- 50-4-60 fue menor en $28.5 \%$ relativo al cemento 100-0-4-60, atribuible únicamente a que el VD no participó tan intensamente en las reacciones de formación de 
Espinoza L. y Escalante J.

productos de hidratación como lo evidencia la presencia de una gran cantidad de granos de VD sin reaccionar o parcialmente reaccionados.

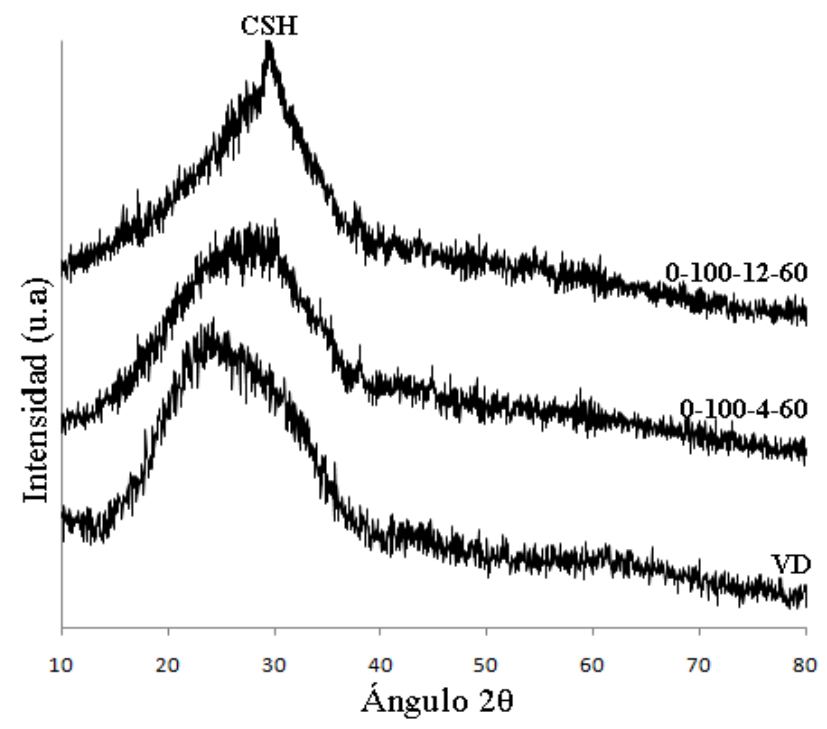

Figura 9. Patrón de DRX del VD sin reaccionar y de cementos 0-100-4-60 y 0-100-12-60

En la Figura 10 B puede notarse variación en el tamaño de los granos de $\mathrm{VD}$, lo que sugiere que la fracción de VD no sometida a AMQ fue la que no participó tan intensamente en las reacciones, disminuyendo la RMC. Al considerar que en el compósito 50-50-4-60 el VD tiene una participación menor en relación a la EAH, se puede pensar que la solución activadora presentó un $\% \mathrm{Na}_{2} \mathrm{O}>4 \%$ con respecto a la EAH. Este incremento en el $\% \mathrm{Na}_{2} \mathrm{O}$ favoreció una mayor reactividad para la $\mathrm{EAH}$, lo cual se puede comprobar al comparar la Figura $10 \mathrm{~A}$ y $\mathrm{B}$, donde se observó que los granos remanentes de EAH fueron de menor tamaño en B como resultado de una reactividad más intensa. Las grietas observadas derivaron del secado de la muestra en la columna del MEB.

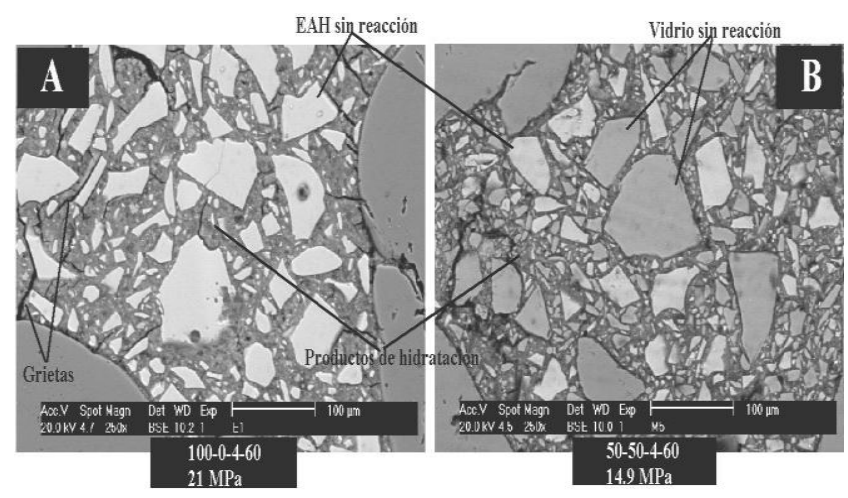

Figura 10. Efecto de la sustitución de EAH por VD
En la Figura $11 \mathrm{~A}$ se presenta la microestructura del cemento 0-100-4-20 (16.1 $\mathrm{MPa}) \quad y$ en $\mathrm{B}$ la microestructura del cemento 0-100-4-60 (16.7 MPa). El mortero curado a $60^{\circ} \mathrm{C}$ se observó más poroso; sin embargo, parece haber un mayor consumo de vidrio. Como era de esperarse la mayor temperatura de curado favoreció la reactividad del vidrio. Este comportamiento ha sido previamente reportado [4] en pastas de EAH activadas con $5 \%$ de $\mathrm{Na}_{2} \mathrm{O}$ con $\mathrm{NaOH}$, se concluyó que a altas temperaturas las reacciones de hidratación ocurren a mayor velocidad; sin embargo, la rápida formación de productos de hidratación impide que estos alcancen a difundir y depositarse en forma homogénea (en contraste con la hidratación a bajas temperaturas) dejando poros en la microestructura. Las similitudes observadas en la RMC entre ambos morteros puede deberse a que en el mortero curado a $20^{\circ} \mathrm{C}$ hay menor avance de las reacciones de formación de productos, pero la matriz se muestra mucho más densa debido a una depositación más homogénea de los productos de hidratación con una menor porosidad.

La Figura 12 A presenta la microestructura de un cemento 0-100-4-60 (16.7 MPa) y B presenta la del cemento 0-100-12-60 (48.1 MPa). Ambas se notaron porosas ya que la alta temperatura de curado aceleró las reacciones y los productos de reacción no se depositaron homogéneamente. Los granos de VD se muestran más reaccionados con $12 \% \mathrm{Na}_{2} \mathrm{O}$ que con $4 \% \mathrm{Na}_{2} \mathrm{O}$ (ataque alcalino es más agresivo). La Figura $\mathrm{B}$ parece indicar que el mecanismo predominante de reacción del VD activado con $12 \% \mathrm{Na}_{2} \mathrm{O}$ y $60^{\circ} \mathrm{C}$ es disolución de la red vítrea (por ataque alcalino) con la consecuente precipitación de productos de carácter cementoso (C-S$\mathrm{H}$ y gel de sílice) en los espacios inicialmente ocupados por el agua. Los granos más pequeños de VD (disminuidos de tamaño por efecto de la AMQ o después de que experimentaron el proceso de disolución sin efecto mecánico) posiblemente reaccionaron por un mecanismo en estado sólido, en el cual los reactantes difunden a través de la capa de productos de hidratación ya formada, consumiendo los granos de VD hacia el interior y precipitando los productos de reacción dentro de las fronteras del grano (anillos de reacción). El mecanismo en estado sólido no se observó en la Figura A ni en los otros cementos 100\% VD. La disminución del $\mathrm{pH}$ por la rápida disolución de las especies químicas ácidas del VD pudo provocar una rápida polimerización del $\mathrm{SiO}_{4}$ disuelto, densificando la microestructura y actuando como barrera para el progreso de las reacciones, por ello tomó lugar el mecanismo topoquímico. 


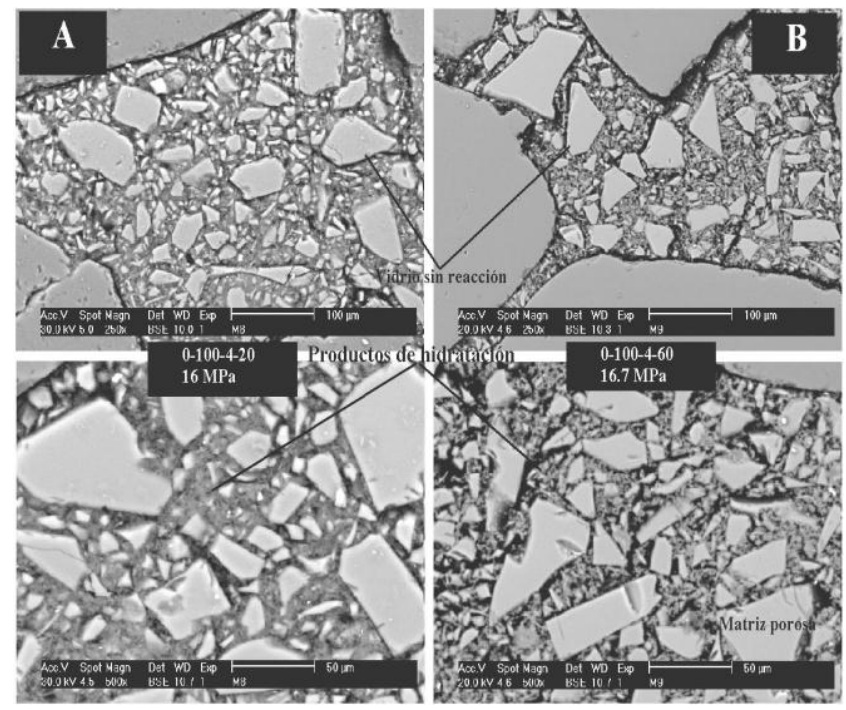

Figura 11. Efecto de la temperatura de curado

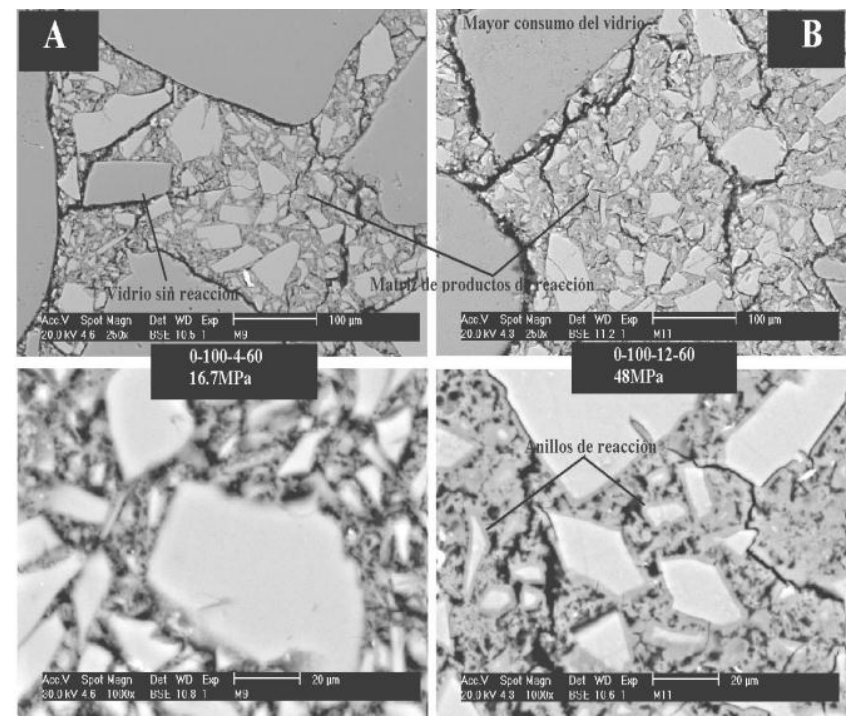

Figura 12. Efecto de la temperatura de curado

\section{Microanálisis puntual}

La Figura 13 presenta los resultados de microanálisis (20 para cada muestra) obtenidos de la matriz de productos de reacción de cementos 100-0-4-60, 75-25-460 y 0-100-4-60. Para el cemento 100-0-4-60 por DRX se discutió que el producto de reacción es un gel C-S-H; las mediciones por EDS indican una relación atómica $\mathrm{Si} / \mathrm{Ca}$ de 0.66 ( \pm 0.074$)$. La relación $\mathrm{Si} / \mathrm{Ca}$ fue mayor que la reportada para $\mathrm{CP}$ hidratado, comúnmente indicada a valores de $\left.0.5{ }^{19}\right]$, pero similar a la reportada por Escalante-García y cols. $\left[{ }^{20}\right]$ para EAH activada por $\mathrm{NaOH}$. Para el cemento 75-25-4-60 la relación atómica $\mathrm{Si} / \mathrm{Ca}$ incrementó a $1.09( \pm 0.16)$. De la Figura 13 es fácil deducir que el incremento de la relación $\mathrm{Si} / \mathrm{Ca}$ al agregar $25 \%$ de VD como cementante se debe a una reducción en el contenido de Ca y a un aumento en el contenido de $\mathrm{Si}$, esto es por el cambio de las proporciones de EAH y VD del compósito. La disolución del VD aporta más Si en solución que se integra en los productos de reacción en dos posibles formas: 1) directamente como parte del $\mathrm{C}-\mathrm{S}-\mathrm{H}, 2)$ precipitando en forma de gel de sílice como ocurre cuando se utiliza silicato de sodio como agente activador; o una suma de 1) y 2). Los análisis puntuales realizados sobre los productos de hidratación del cemento 0- 100-12-60 indican una relación atómica $\mathrm{Si} / \mathrm{Ca}$ de $3.83( \pm 0.62)$; por DRX se identificó gel C-S-H como producto de reacción de este cemento, sin embargo, la alta relación $\mathrm{Si} / \mathrm{Ca}$ sugiere la precipitación de un gel de sílice que favoreció la RMC.

No era de esperarse la formación de un gel geopolimérico (gel NASH, $\mathrm{N}=\mathrm{Na}_{2} \mathrm{O}, \mathrm{A}=\mathrm{Al}_{2} \mathrm{O}_{3}, \mathrm{~S}=\mathrm{SiO}_{2}$, $\mathrm{H}=\mathrm{H}_{2} \mathrm{O}$ ) debido al bajo contenido de $\mathrm{Al}_{2} \mathrm{O}_{3}$ en la composición química del VD $\left(70 \% \mathrm{SiO}_{2}\right.$ y $1.5 \% \mathrm{Al}_{2} \mathrm{O}_{3}$ por FRX). En el caso de la activación química del metacaolín, un silicoaluminato con $57 \% \quad \mathrm{SiO}_{2}$ y $41 \% \mathrm{Al}_{2} \mathrm{O}_{3}$, el principal producto de reacción es el gel $\mathrm{NASH}$, amorfo a la DRX, que presenta estructura tridimensional.

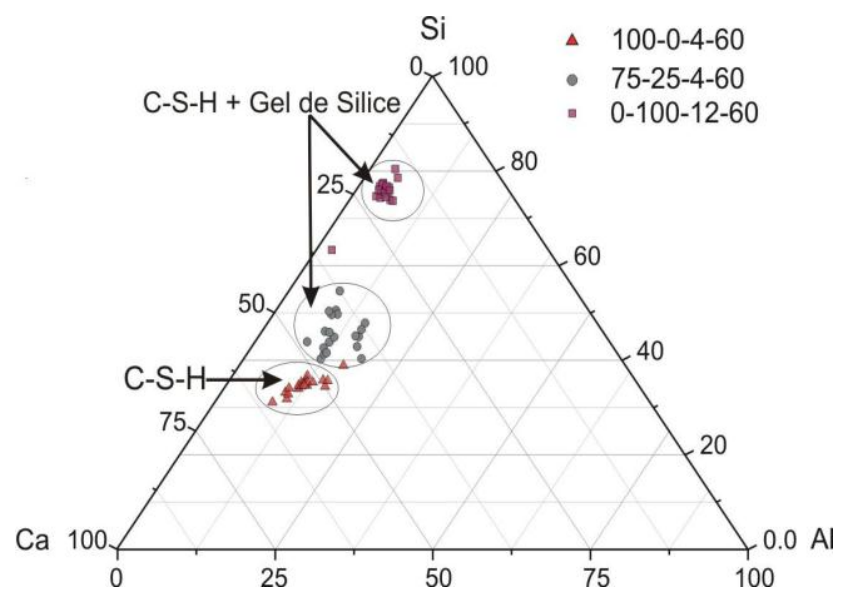

Figura 13. Composición química de la matriz de productos de reacción de cementos 100-0-4-60, 7525-4-60 y 0-100-12-60.

\section{CONCLUSIONES}

El análisis estadístico indicó que en la AMQ las variables de mayor influencia sobre la disolución de la red vítrea del VD fueron: el tiempo de molienda, la relación $\mathrm{Na}_{2} \mathrm{CO}_{3} / \mathrm{NaOH}$ y $\% \mathrm{Na}_{2} \mathrm{O}$. 
Con la AMQ, a mayor concentración $\left(12 \% \mathrm{Na}_{2} \mathrm{O}\right)$ se intensificó el ataque alcalino a la estructura vítrea por el mayor contenido de iones $\mathrm{OH}^{-}$. Sin embargo, se alcanzaron $\mathrm{Ms}$ de 0.047 para $4 \% \mathrm{Na}_{2} \mathrm{O}$ y de 0.029 para $12 \% \mathrm{Na}_{2} \mathrm{O}$.

En los morteros 100\%EAH con activación alcalina convencional, al incrementar el $\% \mathrm{Na}_{2} \mathrm{O}$ se redujo la RMC atribuido a la formación de $\mathrm{Na}_{2} \mathrm{CO}_{3}$ por exceso de álcalis sin reaccionar. También, el aumento en la temperatura de curado de 20 a $60^{\circ} \mathrm{C}$ disminuyó la RMC por la depositación heterogénea de los productos de reacción debido a la rápida disolución de la fracción vítrea, dejando poros en la microestructura.

El principal producto de reacción para los morteros $100 \% \mathrm{EAH}$ fue C-S-H, cuya relación $\mathrm{Si} / \mathrm{Ca}$ incrementó al incrementar la temperatura de curado.

Los morteros 100\%VD presentaron las mayores $\mathrm{RMC}, 48 \mathrm{MPa}$ con $12 \% \mathrm{Na}_{2} \mathrm{O}$ y curado a $60^{\circ} \mathrm{C}$. Los morteros con AMQ 0-100-4-20 y 0-100-4-60 presentaron RMC de aproximadamente $16 \mathrm{MPa}$ a 28 días de curado. El curado a $60^{\circ} \mathrm{C}$ acelera la velocidad de la reacción, sin embargo, a $20^{\circ} \mathrm{C}$ los productos de reacción se depositaron homogéneamente en la microestructura.

Para todos los cementos compósitos, de manera general la RMC se redujo al aumentar el la cantidad de VD. Esto puede atribuirse a la poca reactividad del VD en comparación con la EAH y a que no se alcanzó un Ms suficientemente alto (1-1.5) para una densificación de la microestructura.

En las microestructuras de cementos compósito se observaron granos de VD parcialmente reaccionados, los cuales pueden tener un efecto de relleno mejorando la RMC del compósito.

Los cementos $100 \%$ vidrio tienen excelente potencial para futuras investigaciones por las excelentes propiedades alcanzadas.

\section{REFERENCIAS BIBLIOGRAFICAS}

[1] J. I. Escalante-García, "Materiales alternativos al cemento Portland". Avance y perspectiva, Vol. 21, (2002), pág. 79-88.

$\left.{ }^{2}\right]$ "Fabrican bloques de poroplast". www.elnuevodiario.com.ni/Nacionales/17745

$\left[{ }^{3}\right]$ P. Parameswaran, A. K. Chatterjee, "Alkali activation of Indian blast furnace slag", 8th International congress of chemical cement, Rio de Janeiro, Brazil (1986).

[ $\left.{ }^{4}\right]$ J. I. Escalante-García, J. Méndez-Nonell, A. Gorokhovsky, P. E. Fraire-Luna, H. Mancha-Molinar, G. Mendoza-Suarez, "Reactividad y propiedades mecánicas de escoria de alto horno activada por álcalis", Boletín de la sociedad Española de Cerámica y Vidrio, Vol. 41 (2002) páginas 451 a 458.

[5] J. I. Escalante-García, K. Campos-Venegas, A. Gorokhovsky, A. Fernández, "Cementitious composites of pulverised fuel ash and blast furnace slag activated by sodium silicate: effect of $\mathrm{Na}_{2} \mathrm{O}$ concentration and modulus", Advances in applied ceramics, Vol. 105 (2006) 201-208.

$\left.{ }^{6}\right]$ S. D. Bolboaca, L. Jantschi, "Desing of experiment: Useful orthogonal arrays for number of experiments from 4 to 16", Entropy, Vol. 9 (2007) $198-232$.

[7] I. Turkmen, R. Gul, C. Celik, "A Taguchi approach for investigation of some physical properties of concrete produced from mineral admixtures", Building and Environment 43 (2008) 1127-1137.

$\left[{ }^{8}\right]$ E. Ozbay, A. Oztas, A. Baykasoglu, H. Ozbebek, "Investigating mix proportions of high strength self compacting concrete by using Taguchi method", Construction and building materials 23 (2009) 694702.

[9] O. Tan, A. S Zaimoglu, S. Hinislioglu, S. Altun, "Taguchi approach for optimization of the bleeding on cement-based grouts", Tunnelling and underground space technology 20 (2005) page 167173. 
$\left[{ }^{10}\right]$ A. Fernández-Jiménez, A. Palomo, M. Criado, "Activación alcalina de cenizas volantes. Estudio comparativo entre activadores sódicos y potásicos". Materiales de construcción, Vol. 56 (2006) page 281, 51-65.

$\left[{ }^{11}\right]$ Wang S. D, Scrivener K. L, Pratt P. L, "Factors affecting the strenght of alkali-activated slag". Cement and concrete research (1994) 24:1033.

$\left[{ }^{12}\right]$ O. Burciaga-Díaz, J. I. Escalante-García, R. Arellano-Aguilar and A. Gorokhovsky, "Statistical analysis of strength development as a function of various parameters on activated metakaolin/slag cements", J of the American Ceramic Society 93 (2) (2010) page 541-547.

$\left[{ }^{13}\right]$ J. W. Phair, J. S. J Deventer, J. D. Smith, "Mechanism of polysialation in the incorporation of zirconia into fly ash-based geopolymers", Ind. Eng. Chem. Res. 39 (2000) page 2925-2934.

$\left[{ }^{14}\right]$ C. K. Yip, J. S. J. Van Deventer, "Microanalysis of Calcium silicate hidrate gel formed within a geopolymeric binder", J. Mater. Sci. 38 (2003) page 3851-3860.

[15] M. Criado, A. Fernández-Jiménez, A. G. de la Torre, A. Palomo, G. López-Olmo, M. M. Alonso, M. A. G. Aranda, "An XRD study of the effect of the $\mathrm{SiO} 2 / \mathrm{Na} 2 \mathrm{O}$ ratio on the alkali activation of fly ash". Cement and concrete research 37, (2007) page 671679.

[16] M. Monzó, A. Fernández-Jiménez, M. Vicent, A. Palomo, A. Barba, "Activación alcalina de metacaolín. Efecto de la adición de silicato soluble y de la temperatura de curado", Bol Sociedad Española de Cerámica y Vidrio, V. 47, 1, (2008) page 35-43

[17] R. Arellano-Aguilar, O. Burciaga-Díaz, J. I. Escalante-García, "Lightweight concretes of activated metakaolin-fly ash binders, with blast furnace slag aggregates", Construction and building materials 24 (2010) page 1166-1175.
[18] A. R. Sakulich, "Characterization of environmentally -friendly alkali activated slag cement and ancient building materials", Ph.D Thesis, Drexel University, 2009.

[19] I. Lecomte et al. "(Micro)-Structural comparison betwen geopolymers, alkali-activated slag cement and Portland cement", Journal of the European ceramic society 26 (2006) page 3789-3797.

[20] J. I. Escalante-García, P. E. Fraire-Luna, Antonio F. Fuentes, Alexander Gorokhovsky and Guillermo Mendoza-Suarez, "Hydration products and reactivity of blast furnace slag activated by various alkalis", J. Am. Ceram. Soc 86 [12] (2003) page 2148-53.

\section{AGRADECIMIENTOS}

Los autores agradecen al CONACYT de México por el financiamiento del proyecto de investigación 53563, a la SRE de México y a la UNI de Nicaragua por la beca otorgada a L. J. Espinoza-Pérez para obtener el grado de Maestro en Ciencias.

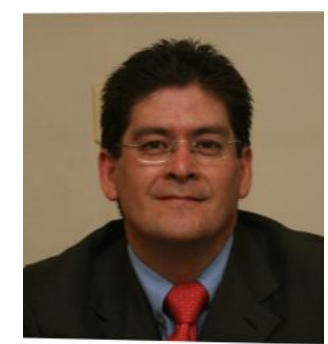

El Doctor J. Iván Escalante es Profesor-Investigador titular de CINVESTAV-IPN. Su área de interés es el desarrollo de materiales cementosos y cerámico-cementosos a partir de desechos inorgánicos.

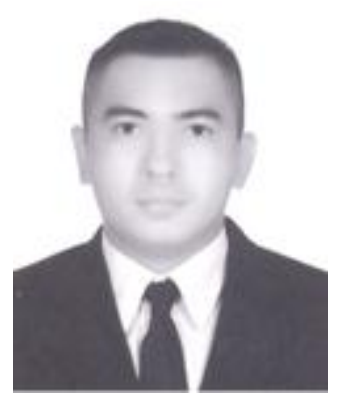

El Maestro Léster Espinoza es docente de la Facultad de Ingeniería Química de la UNI. Su área de interés es la química de hidratación y propiedades físico-mecánicas de materiales de construcción alternativos. 